DOI 10.12737/article_5cceddbb89a966.13019711

УДК $621.372 .413 ; 631.363$

ГРАНУЛИРОВАНИЕ ОТХОДОВ СЫРЬЯ

В ПРОЦЕССЕ ДИЭЛЕКТРИЧЕСКОГО НАГРЕВА

Крайнов Ю.Е., Коробков А.Н., Белова М.В., Новикова Г.В.

Реферат. Целью работы является разработка и обоснование параметров установки для гранулирования непищевых отходов животного и растительного происхождения в процессе диэлектрического нагрева. Задачи исследования: разработать микроволновые установки с объемными резонаторами разной конфигурации, обеспечивающие высокую напряженность электрического поля и непрерывность процесса гранулирования сырья; вычислить параметры электродинамической системы генератора (напряженность электрического поля, собственная добротность резонатора) и визуализировать картину распределения электромагнитного поля в резонаторах разной конфигурации. В исследованиях базировались на теории диэлектрического нагрева и теория формования гранул из многокомпонентного сырья. Исследование параметров электродинамической системы сверхвысокочастотного генератора проводили в программе CST Microwave Studio. Проанализированы конструкционные исполнения объемных резонаторов с точки зрения реализации требований, предъявляемых сверхвысокочастотным установкам и грануляторам, предназначенным для использования в фермерских хозяйствах. Анализированы разработанные установки с тороидальным резонатором и дисковой матрицей и с эллипсоидными резонаторами и зубчатыми кольцевыми матрицами. Визуализированы распределения электромагнитного поля в разработанных конструкционных исполнениях резонаторов, совмещенных с рабочей камерой грануляторов. Вычислены величины собственной добротности резонаторов и напряженности электрического поля в них.

Ключевые слова: сверхвысокочастотный генератор, резонатор, гранулятор, матрица, отходы сырья, эффективные режимы.

Введение. Министерством промышленности и торговли РФ технологии создания изделий сверхвысокочастотной электроники отнесены к критичным технологиям (стратегическая программа от 17.12.2012 г) [1]. СВЧ установки отличаются по физическому принципу действия и конструкции. За рубежом разрабатывают СВЧ установки на основе источников, мощностью от 25 до 50 кВт, с водяным охлаждением, при этом достичь равномерного нагрева сырья сложно. Стоимость установленной мощности оценивается до 3 тыс. долларов за 1 кВт. Анализ разработок производителей СВЧ установок в России позволяет выявить основные тенденции и перспективы развития устройств различного функционального назначения, в том числе для гранулирования сырья. Поэтому для реализации необходимых электродинамических и эксплуатационных характеристик нами разрабатываются новые конструкции объемных резонаторов с маломощными магнетронами и способы, совмещающие микроволновую технологию с процессом гранулирования многокомпонентного сырья. Это позволит улучшить физико-механические свойства гранул.

Условия, материалы и методы исследований. В исследованиях базировались на теории диэлектрического нагрева и теории формования гранул из многокомпонентного сырья. Исследование параметров электродинамической системы сверхвысокочастотного генератора проводили в программе CST Mi- crowave Studio [2]. Оценка технических характеристик установки проводилась с помощью современных цифровых приборов и методик. Трехмерные моделирования конструктивного исполнения СВЧ установок выполнены в программе Компас-3D V17.

Анализ и обсуждение результатов исследований. Разработаны и анализированы конструкционные исполнения объемных резонаторов с точки зрения реализации требований, предъявляемых установкам с источниками энергии электромагнитных излучений и грануляторам, предназначенным для использования в фермерских хозяйствах. Ниже описаны разработанные две установки: 1) с тороидальным резонатором и дисковой матрицей; 2) с эллипсоидными резонаторами и зубчатыми кольцевыми матрицами.

1. Установка с тороидальным резонатором и дисковой матрицей для гранулирования обезвоженных непищевых отходов убоя животных в процессе диэлектрического нагрева.

Известны квазистационарные резонаторы, у которых резко выраженное пространственное разъединение электрического и магнитного полей, что позволяет рассматривать такие резонаторы как соединение сосредоточенных емкости и индуктивности [3, 4]. В тороидальном резонаторе, в малом зазоре между стенками резонатора сосредоточена энергия электрического поля. Этот зазор может рассматриваться как плоский конденсатор. На этом принципе построена конфигурация предлагае- 
мого тороидального резонатора при сохранении достаточно большого объема и его собственной добротности.

Рабочая камера установки (рисунок 1) представлена в виде тороидального резонатора 1 с прямоугольным сечением тора. Тороидальный резонатор выполнен из коаксиально расположенных неферромагнитных цилиндров 2,3, расположенных вертикально и имеющих разную высоту. Нижним основанием внутреннего цилиндра 3 с меньшей высотой служит неферромагнитная решетка 11 от измельчителя. Функцию нижнего основания наружного цилиндра 2 выполняет вращающаяся от мотора-редуктора матрица 5 из неферромагнитного материала с множеством конусообразных отверстий [5]. В пространстве между решеткой и вращающейся матрицей расположены прижимные диэлектрические ролики 6 с ребристой поверхностью. Они прижаты к вращающейся матрице за счет специального винта, позволяющего регулировать расположения диэлектрической оси роликов. Верхнее основание наружного цилиндра 2 закрыто кольцевой поверхностью. Во внутреннем цилиндре расположен нагнетательный шнек 10, вращающийся нож 9 измельчителя, а решетка является основанием. Измельчитель имеет лоток для приема сырья. СВЧ генераторы 4 вводят энергию в резонатор из тороидальной части, т.е. магнетроны и волноводы расположены на образующей наружного цилиндра. Под вращающейся матрицей 5 имеется нож 12 , расположенный стационарно, для срезания гранул, которые выводятся за пределы установки через отверстие выгрузного корпуса. Вращение матрицы осуществляется с помощью мотора-редуктора. Под вращающейся матрицей имеется выгрузной корпус с отверстием.

Диаметр матрицы (диска) должен соответствовать диаметру наружного цилиндра 2 . Зазор между наружным цилиндром и матрицей минимальный. Имеется несколько съемных матриц с определенным диаметром отверстий для разного вида сырья. Отверстия имеют форму конуса, чтобы сырье плотнее сжималась. Толщина матрицы не менее 2 см. Для эффективной работы тороидального резонатора зазор между матрицей 5 и решеткой 11 должен быть меньше радиуса внутреннего цилиндра 3. Все размеры тороидального резонатора согласованы с длиной волны. При этом применять объемные резонаторы, размеры которых на много велики по сравнению с длиной волны, нецелесообразно из-за возбуждения в них большого числа колебаний. Поэтому проектируется резонатор, максимальные размеры которого не превышают 3 - 5 длины волны.

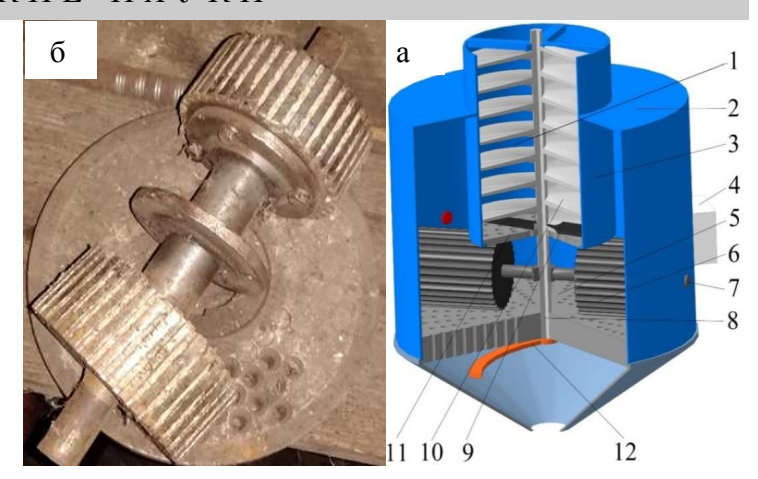

1 - тороидальный резонатор в виде коаксиально расположенных наружного 2 и внутреннего 3 цилиндров; 4 - СВЧ генераторы; 5 - дисковая матрица;

6 - прижимные диэлектрические ролики; 7 - ось; 8 - вал от мотора-редуктора; 9 - вращающийся нож; 10 - нагнетательный шнек измельчителя; 11 - неферромагнитная решетка; 12 - стационарный нож

Рисунок 1 - Установка с тороидальным резонатором для гранулирования обезвоженных непищевых отходов убоя животных в ЭМПСВЧ: а) пространственное изображение; б) матрица и ролики гранулятора базового варианта;

Технологический процесс термообработки и гранулирования сырья происходит следующим образом. Включают мотор-редуктор для вращения матрицы 5. Включают электропривод измельчителя, на приемный лоток которого подают предварительно подготовленное сырье, влажностью 16...18\%. Включают СВЧ генераторы 4. Сырье с помощью нагнетательного шнека 10 и вращающегося ножа 9 перемешивается, измельчается и выдавливается через решетку в пространство, конденсаторную часть тороидального резонатора. Здесь измельченное сырье подвергается воздействию электромагнитного поля сверхвысокой частоты (ЭМПСВЧ), эндогенно нагревается, т.е. происходит термическая обработка сырья и, следовательно, обеззараживание продукта. При вращении матрицы, прижатые к ней диэлектрические ролики, расположенные на оси 7, начинают также вращаться. С помощью прижимных роликов, нагретое сырье продавливается через конусообразные отверстия в матрице 5. Спрессованная масса, проходя сквозь отверстия матрицы, нагревается дополнительно, принимает форму длинного цилиндра, разрезается стационарным ножом 12 на небольшие гранулы. Через отверстие в выгрузной емкости готовые гранулы высыпаются в тару. В случае перемешивания боенских отходов с отходами злаковых культур, крахмал в процессе термической обработки превращается в сахар, а это повышает кормовую ценность продукта.

2. Установка с эллипсоидными резонаторами и зубчатыми кольцевыми матрицами для гранулирования сырья в процессе диэлектрического нагрева. 
Известна установка для гранулирования измельченного сырья с зубчатыми кольцевыми матрицами с двумя равными колесамиматрицами, имеющими внешнее зацепление $[5,6]$. Этот гранулятор выдавливающего типа с формированием гранул в канале прессования кольцевой матрицы. В них сырье, попадая в полость между колесами-матрицами, вдавливается зубьями в канал прессования. Выдавливаемые из канала прессования гранулы диаметром 10 - 14 мм срезаются неподвижным ножом. Такие грануляторы не могут обеспечить высокую прочность и плотность гранул, и обеззараживать их в связи с короткой выдержкой сырья под давлением; необходимо создавать высокое давление для сдвига сжатого сырья в матричных каналах; существенный нагрев матриц при сдвиге частиц сырья и потери этого тепла в окружающую среду [5].

Установка для гранулирования измельченного сырья с зубчатыми кольцевыми матрицами в процессе диэлектрического нагрева содержит эллипсоидные резонаторы 1 и зубчатые кольцевые матрицы 4 (рисунок 2). Внутри каждого из двух эллипсоидных резонаторов, выполненных из равных нижних и верхних сферических частей, расположены зубчатые кольцевые матрицы из диэлектрического материала. Они установлены между прижимными диэлектрическими кольцами 13. Верхние кольца жестко закреплены к валам, один из которых приводной 7, а второй ведомый 11 . По наружным периметрам зубчатых кольцевых матриц, до зоны внешнего зубчатого зацепления 9, установлены экранирующие ободы 3 , имеющие внутренние пазы для зубьев. В нижних частях эллипсоидных резонаторов имеются выгрузные кольцевые отверстия 8, шириной не более, чем четверть длины волны, и диэлектрические скребки 6 , прикрепленные к валам. В верхних частях эллипсоидных резонаторов расположены излучатели 2 от СВЧ генераторов. На уровне каналов для прессования 10, с внутренней стороны зубчатых кольцевых матриц 4, жестко закреплен диэлектрический нож 5 к нижнему основанию приемного лотка 12 из неферромагнитного материала, установленного под эллипсоидными резонаторами.

Камера для приема измельченного сырья 14 , выполненная из неферромагнитного материала, расположенная над эллипсоидными резонаторами, на нижнем основании, в зоне внешнего зубчатого зацепления 9, имеет прорезь. Установка содержит две одинаковые зубчатые кольцевые матрицы 4, одна из которых является ведущей, а другая ведомой. Матрицы выполнены с каналами прессования во впадинах. Поэтому конструкционнотехнологическую схему установки можно рас-

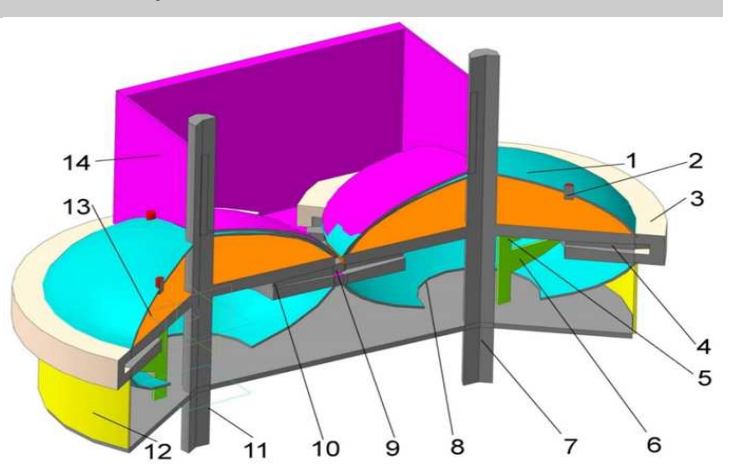

Рисунок 2 - Установка с зубчатыми кольцевыми матрицами и эллипсоидными резонаторами для гранулирования измельченного сырья:

1 - эллипсоидные резонаторы; 2 - излучатели от СВЧ генераторов; 3 - экранирующий обод;

4 - зубчатые кольцевые матрицы из фторопласта;

5 - нож для срезания гранул; 6 - скребок для выгрузки

гранул; 7 - приводной вал; 8 - выгрузное кольцевое

отверстие; 9 - внешнее зубчатое зацепление; 10 - каналы для прессования в матрице;

11 - ведомый вал; 12 - приемный лоток для гранул; 13 - прижимные кольца;

14 - камера для приема измельченного сырья

сматривать как пресс с выдавливающими одинаковыми зубчатыми кольцевыми матрицами внешнего зацепления.

Рабочий процесс происходит следующим образом. Смешанное сырье определенной влажности $(20-25 \%)$ из камеры 14 для приема измельченного сырья попадает на зубчатые кольцевые матрицы 4. Так как приводная матрица вращается от электродвигателя, то вторая ведомая матрица за счет зацепления ее зубьев с зубьями приводной матрицы тоже вращается. Тогда сырье поступает в пространство между зубьями 9 и их поверхностью вдавливается в каналы прессования 10 в матрицах. По мере движения сырья в канале прессования напряжения в гранулах уменьшается, осуществляется релаксация напряжений и к выходу она упрочняется [7]. Образованные гранулы вытесняются вновь поступающими порциями сырья и на выходе из каналов прессования срезаются диэлектрическим ножом 5 и падают в лоток для приема гранул 12. Далее гранулы с помощью диэлектрического скребка 6 выгружаются через кольцевое отверстие 8. Сырье, выброшенный из пространства между зубами, попадает на основание камеры 14 для приема измельченного сырья, откуда в последующем попадает в рабочий процесс.

По геометрии зацепления зубьев кольцевой матрицы установка имеет весьма жесткие зацепления, так как диаметры кольцевых матриц равны. Поэтому каналы прессования можно использовать в пределах короткой дуги окружности кольца. Для удержания сырья на рабочей поверхности зубьев целесообразно располагать кольцевые матрицы в горизонтальной плоскости, а подачу сырья осуществ- 
Таблица - Технические характеристики установок, обеспечивающих гранулирования сырья в процессе диэлектрического нагрева

\begin{tabular}{|c|c|c|}
\hline \multirow{2}{*}{ Характеристики } & \multicolumn{2}{|c|}{ Микроволновая установка с } \\
\cline { 2 - 3 } & $\begin{array}{c}\text { плоской } \\
\text { матрицей }\end{array}$ & зубчатыми кольцевыми матрицами \\
\hline Производительность установки, кг/ч & $182-194$ & 150 \\
\hline Мощность СВЧ генераторов, кВт & 3,6 & 3,6 \\
\hline Мощность электродвигателя, кВт & 7,5 & 5,5 \\
\hline Частота вращения матрицы, об/мин & 300 & 155 \\
\hline Диаметр вальцов, мм & 200 & 200 \\
\hline Диаметр отверстий в матрице, мм & 4 & 8 \\
\hline Габаритные размеры, мм & $950 \times 400 \times 600$ & $950 \times 1200 \times 16000$ \\
\hline Масса, кг & 110 & 185 \\
\hline
\end{tabular}

лять сверху. Плотность и площадь поперечного сечения гранул можно регулируют с учетом зоотехнических требований. Количество каналов прессования 10 в зубчатых кольцевых матрицах ограничивается по условиям надежности и технологии его изготовления, например из фторопласта. Минимальная длительность нахождения сжатого сырья в каналах прессования определяется исходя из его упруго-вязких свойств и удельной мощности СВЧ генератора. Длину канала прессования следует выбирать по условиям необходимого противодавления и релаксации напряжений в грануле. Коэффициент использования площади канала прессования учитывает эффективность процесса подачи сырья в зону сжатия и степень заполнения объема пространства между зубьями. На увеличения пропускной способности установки влияет количество каналов прессования и их поперечное сечение, частота вращения зубчатых кольцевых матриц [8].

Основная задача при проектировании таких установок - это правильное согласование параметров электродинамической системы генератора, а именно величину напряженности электрического поля с собственной добротностью и объемом резонатора, далее мощностью генератора и диэлектрическими параметрами многокомпонентного сырья $[9,10]$.

Во время разработки резонаторов решающим является равномерное распределение электромагнитного поля в нем. Поэтому вычисления и визуализация распределения электромагнитного поля в разработанных объемных резонаторах проводили в режиме переходного процесса в программе CST Microwave Studio [2]. Данная программа позволяет для разной конфигурации резонаторов вычислить и визуализировать распределения напряженности электромагнитного поля, плотности тока и добротность резонатора. Далее можно обосновать конфигурацию и объем резонатора [11].

В процессе проектирования объемных резонаторов в трехмерном представлении с по- мощью указанной программы задаются граничные условия, и определяется местоположение источников излучения [12]. Далее выполняется разбиение всего пространства задачи на сетку, а затем рассчитывается электрическое и магнитное поле в каждой точке пространства [13]. Такое компьютерное моделирование позволило проанализировать и оптимизировать поведение отдельных узлов гранулятора с источником СВЧ энергии на стадии проектирования.

Технические характеристики установок, обеспечивающих гранулирование сырья в процессе диэлектрического нагрева, приведены в табл.

Выводы и рекомендации. Анализ разработанных установок показывает, что установку с тороидальным резонатором и дисковой матрицей рекомендуется использовать для гранулирования обезвоженных непищевых отходов убоя животных при высокой напряженности электрического поля, обеспечивающей снижение бактериальной обсемененности сырья. Достоинства: не большая скорость вращения матрицы, следовательно, меньший износ роликов, простота в обслуживании.

Установку с эллипсоидными резонаторами и зубчатыми кольцевыми матрицами рекомендуется использовать для производства стерильных гранул высокого качества из отходов растительного и животного происхождения, предварительно смешенного в определенной концентрации. Установка имеет низкую производительность, но рабочие органы долговечны.

При производительности гранулятора $182 \ldots 194$ кг/ч и удельной мощность СВЧ генератора $1,4 \ldots 2$ Вт/г продолжительность воздействия ЭМПСВЧ на сырье массой $0,8 \ldots 1$ кг в резонаторной камере должна составлять $10 \ldots 12$ с. Это обеспечивает минимальные удельные энергетические затраты 0,06 кВт·ч/ кг.

Повышения прочности гранул достигается за счет избирательного диэлектрического нагрева многокомпонентного сырья. 
1. hse.ru>...2013/02/12...СВЧ технологии 2512 2012.pdf. Стратегическая программа исследований технологической платформы «СВЧ технологии».

2. Курушин А.А., Пластиков А.Н. Проектирование СВЧ устройств в среде CST Microwave Studio. М.: МЭИ, 2011. - 155 с..

3. Стрекалов, А.В. Электромагнитные поля и волны / А.В. Стрекалов, Ю.А. Стрекалов. - М.: РИОР: ИНФРА-М, 2014. - 375 с.

4. Дробахин, О.О. Резонансные свойства микроволновых резонаторов в виде сферического сектора / О.О. Дробахин, П. И. Заболотный, Е. Н. Привалов // Радиоэлектроника. Информатика. Управление. 2009. - № 2(21). - С. 11-16.

5. Булатов И.А. Разработка процесса прессового гранулирования мелкодисперсных сред на примере минеральных порошков и древесных отходов: автореф. ...канд. техн. наук: 05.17.08, 05.02.13. - М., 2012. $25 \mathrm{c}$.

6. Маврин, О. Оптимальное решение: гранулирование и термообработка за 240 секунд / Маврин О. // Комбикорма. - 2009. - № 1. - С. 33-35.

7. Макаренков, Д.А. Особенности выбора гранулирующего оборудования многокомпонентных полидисперсных шихт, с вторичными материальными ресурсами на основе системного анализа / Д.А. Макаренков, В.И. Назаров, А.М. Гонопольский, Я.А. Трефилова // Вестник МГОУ. Серия «Естественные науки». - 2013. - № 1. - С. 49-64.

8. Афанасьев, В.А. Разработка технологии влажного прессования углеводно-витаминно-минеральных добавок с повышением содержания мелассы / В.А. Афанасьев, А.А. Киселев // Вестник Воронежского государственного университета инженерных технологий. - 2015. - № 1 (63). - С.70-73.

9. Жданкин, Г.В. Анализ разработанных сверхвысокочастотных установок для термообработки сырья / Г.В. Новикова, Г.В. Жданкин, О.В. Михайлова, А.А. Белов // Вестник Казанского государственного аграрного университета. - 2016. - № 4 (42). - С. 89-93.

10. Жданкин, Г.В. Разработка и обоснование параметров установки для диэлектрического нагрева непищевых отходов животного происхождения в непрерывном режиме / Г.В. Жданкин, Г.В. Новикова, О.В. Михайлова, Н.К. Кириллов // Вестник НГИЭИ. - 2017. - № 2(69). - С. 61-71.

11. Zhdankin, G.V. Microwave installation with conical resonators for the heat treatment of inedible meat wastes / O.V. Mikhailova, Semenov V. G., Baimukanov D. A., Iskhan K. Zh., Kalagambet M. B., Nurbaev S. D., H. A. Aubakirov // Of the National academy of sciences of the republic of Kazakhstan [Series of geology and technical sciences] .- Volume 2, Number 428.- 2018, pp. 44-54.

12. Zhdankin, G.V. Installation for complex effects electrophysical factors on raw // O.V. Mikhailova // Of the National academy of sciences of the republic of Kazakhstan [Series of geology and technical sciences].- Volume 1, Number 428.- 2019, pp. 36-46.

13. Shamin, E.A. Microwave technologies and devices separating fur from rabbit skin /O.V. Mikhailova, I.G. Ershova, D.V. Poruchikov, S.P. Zaycev // Amazonia Investiga. - Volume 7, Number 17. - 2018, pp. 630-640.

Сведения об авторах:

Крайнов Юрий Евгеньевич - преподаватель кафедры «Электрификация и автоматизация», e-mail: krainov24@mail.ru

Коробков Алексей Николаевич - преподаватель кафедры «Электрификация и автоматизация»

Белова Марьяна Валентиновна - доктор технических наук, доцент кафедры «Электрификация и автоматизация»

Новикова Галина Владимировна - доктор технических наук, профессор, главный научный сотрудник, еmail: NovikovaGalinaV@yndex.ru

ГБОУ ВО «Нижегородский инженерно-экономический университет», Нижегородская область, г. Княгинино, Россия.

\section{GRANULATION OF RAW MATERIALS DURING THE DIELECTRIC HEATING PROCESS}

Kraynov Yu.E., Korobkov A.N., Belova M.V., Novikova G.V.

Abstract. The aim of the work is to develop and justify the parameters of the installation for the granulation of non-food waste of animal and vegetable origin in the process of dielectric heating. Objectives of the study: to develop microwave installations with volumetric resonators of different configurations, providing high electric field strength and continuity of the process of granulating raw materials; calculate the parameters of the electrodynamic system of the generator (electric field strength, the intrinsic $\mathrm{Q}$ factor of the resonator) and visualize the distribution pattern of the electromagnetic field in the resonators of different configurations. The studies were based on the dielectric heating theory and the theory of forming granules from multicomponent raw materials. The parameters of the electrodynamic system of the microwave generator were studied in the CST Microwave Studio program. The structural performance of volume resonators is analyzed from the point of view of the implementation of the requirements imposed by microwave devices and granulators intended for use in farms. Analyzed devices with a toroidal resonator and a disk matrix and with ellipsoid resonators and gear ring matrices are analyzed. The electromagnetic field distributions are visualized in the developed design versions of resonators combined with the working chamber of granulators. The values of the self-quality of the resonators and the electric field strength in them are calculated.

Key words: microwave generator, resonator, granulator, matrix, waste materials, effective modes. 


\section{References}

1. hse.ru>...2013/02/12...SVCh tekhnologii 25.12.2012.pdf. Strategicheskaya programma issledovaniy tekhnologicheskoy platformy "SVCh tekhnologii". (Microwave Technology 25.12.2012.pdf. Strategic research program of the technological platform "Microwave technology").

2. [http://www.eurointech.ru/], Kurushin A.A., Plastikov A.N. Proektirovanie SVCh ustroystv v srede CST Microwave Studio. (Designing microwave devices in the CST Microwave Studio environment). - M.: MEI, 2011. - P. 155.

3. Strekalov A.V. Elektromagnitnye polya i volny. [Electromagnetic fields and waves]. / A.V. Strekalov, Yu.A. Strekalov. - M.: RIOR: INFRA-M, 2014. - P. 375.

4. Drobakhin O.O. Resonance properties of microwave resonators in the form of a spherical sector. [Rezonansnye svoystva mikrovolnovykh rezonatorov v vide sfericheskogo sektora]. / O.O. Drobakhin, P.I. Zabolotnyy, E.N. Privalov // Radioelektronika. Informatika. Upravlenie. - Radioelectronics. Computer science. Control. 2009. - № 2(21). - P. 11-16.

5. Bulatov Ilya Anatolevich. Razrabotka protsessa pressovogo granulirovaniya melkodispersnykh sred na primere mineralnykh poroshkov i drevesnykh otkhodov: avtoref. ...kand. tekhn. nauk: 05.17.08, 05.02.13. (Development of the process of press granulation of finely dispersed media on the example of mineral powders and wood waste: author's abstract for a degree of Ph.D. of Technivs: 05.17.08, 05.02.13). - M., 2012. - P. 25.

6. Mavrin O. Optimal solution: granulation and heat treatment for 240 seconds. [Optimalnoe reshenie: granulirovanie i termoobrabotka za 240 sekund]. / Mavrin O. // Kombikorma. - Combined feed. - 2009. - № 1. - P. 33-35.

7. Makarenkov D.A. Features of the choice of granulating equipment multicomponent polydisperse charges, with secondary material resources based on system analysis. [Osobennosti vybora granuliruyuschego oborudovaniya mnogokomponentnykh polidispersnykh shikht, s vtorichnymi materialnymi resursami na osnove sistemnogo analiza]. / D.A. Makarenkov, V.I. Nazarov, A.M. Gonopolskiy, Ya.A. Trefilova // Vestnik MGOU. Seriya "Estestvennye nauki". - The herald of MGOU. Series "Natural Sciences". - 2013. - №1. - P. 49-64.

8. Afanasev V.A. Development of technology for wet pressing carbohydrate-vitamin-mineral supplements with an increase in the content of molasses. [Razrabotka tekhnologii vlazhnogo pressovaniya uglevodno-vitaminno-mineral'nykh dobavok s povysheniem soderzhaniya melassy]. / V.A. Afanasev, A.A. Kiselev // Vestnik Voronezhskogo gosudarstvennogo universiteta inzhenernykh tekhnologiy. - The Hreald of Voronezh State University of Engineering Technologies. 2015. - № 1 (63). - P. 70-73.

9. Zhdankin G.V. Analysis of developed microwave installations for heat treatment of raw materials. [Analiz razrabotannykh sverkhvysokochastotnykh ustanovok dlya termoobrabotki syrya]. / G.V. Novikova, G.V. Zhdankin, O.V. Mikhaylova, A.A. Belov // Vestnik Kazanskogo gosudarstvennogo agrarnogo universiteta. - The Herald of Kazan State Agrarian University. - Kazan: FGBOU VO Kazanskiy GAU. 2016, № 4 (42). - P. 89-93.

10. Zhdankin G.V. Development and justification of the parameters of the installation for dielectric heating of nonfood waste of animal origin in a continuous mode. [Razrabotka i obosnovanie parametrov ustanovki dlya dielektricheskogo nagreva nepischevykh otkhodov zhivotnogo proiskhozhdeniya v nepreryvnom rezhime]. / G.V. Zhdankin, G.V. Novikova, O.V. Mikhaylova, N.K. Kirillov // - Knyaginino: Vestnik NGIEI. - The Herald of NIEEI. № 2(69), 2017. - P. $61-71$.

11. Zhdankin, G.V. Microwave installation with conical resonators for the heat treatment of inedible meat wastes / O.V. Mikhailova, Semenov V. G., Baimukanov D. A., Iskhan K. Zh., Kalagambet M. B., Nurbaev S. D., H. A. Aubakirov // Of the National academy of sciences of the republic of Kazakhstan [Series of geology and technical sciences] .Volume 2, Number 428.- 2018, pp. 44-54.

12. Zhdankin, G.V. Installation for complex effects electrophysical factors on raw // O.V. Mikhailova // Of the National academy of sciences of the republic of Kazakhstan [Series of geology and technical sciences].- Volume 1, Number 428. 2019, pp. 36-46.

13. Shamin, E.A. Microwave technologies and devices separating fur from rabbit skin / O.V. Mikhailova, I.G. Ershova, D.V. Poruchikov, S.P. Zaycev // Amazonia Investiga. - Volume 7, Number 17. - 2018, pp. 630-640.

Authors:

Krainov Yuriy Evgenevich - Lecturer of Electrification and Automation Department, E-mail krainov24@mail.ru Korobkov Aleksey Nikolaevich - Lecturer of Electrification and Automation Department, el. 89200148021.

Belova Maryana Valentinovna - Doctor of Technical Sciences, Associate Professor of Electrification and Automation Department

Novikova Galina Vladimirovna - Doctor of Technical Sciences, Professor, Chief Researcher, NovikovaGalinaV@yndex.ru

Nizhny Novgorod Engineering and Economic University, Knyaginino, Nizhny Novgorod Region, Russia. 